\title{
BMJ Open Protocol for a systematic review and meta-analysis of interventions for pathologic perforator veins in chronic venous disease
}

\author{
Melissa Andreia de Moraes Silva, ${ }^{\oplus 1,2}$ Ronald Luiz Gomes Flumignan, ${ }^{\odot 1}$ \\ Fausto Miranda, ${ }^{\oplus 1}$ Rodolfo Souza Cardoso, ${ }^{\oplus 2}$ Seleno Glauber de Jesus Silva, 2 \\ Henrique Jorge Guedes, ${ }^{\oplus}$ Luis Carlos Uta Nakano ${ }^{\oplus}$
}

To cite: de Moraes Silva MA, Flumignan RLG, Miranda F, et al. Protocol for a systematic review and meta-analysis of interventions for pathologic perforator veins in chronic venous disease. BMJ Open 2019;9:e024088. doi:10.1136/ bmjopen-2018-024088

- Prepublication history for this paper is available online. To view these files please visit the journal online (http://dx.doi. org/10.1136/bmjopen-2018024088).

MAMS and RLGF contributed equally.

Received 9 May 2018

Revised 4 March 2019

Accepted 8 March 2019

Check for updates

(c) Author(s) (or their employer(s)) 2019. Re-use permitted under CC BY-NC. No commercial re-use. See rights and permissions. Published by BMJ.

${ }^{1}$ Department of Surgery, Division of Vascular and Endovascular Surgery, Universidade Federal de São Paulo, São Paulo, SP, Brazil

${ }^{2}$ Division of Vascular and Endovascular Surgery, Hospital de Clínicas de Itajubá, Itajubá, MG, Brazil

\section{Correspondence to}

Professor Ronald Luiz

Gomes Flumignan;

flumignan@gmail.com

\section{ABSTRACT}

Introduction Perforator veins (PVs) play an important role in the development of chronic venous insufficiency and ulceration. Procedures to eliminate incompetence and reflux in PV may include open surgery, subfascial endoscopic surgery, intravenous ablation techniques and sclerotherapy. With the aim of filling the evidence gap, this is a protocol for a systematic review that will assess the effects of any form of intervention for the treatment of pathologic PVs of the lower limbs in patients with chronic venous disease.

Methods and analysis Systematic searches will be carried out in MEDLINE, EMBASE, Cochrane CENTRAL, IBECS and LILACS databases at a minimum without date or language restrictions for relevant randomised controlled trials (RCTs) and quasi-RCTs (trials in which the method of allocation is not truly random). In addition, a search will also be carried out in the WHO International Clinical Trials Registry Platform, in the clinical trial registries of ClinicalTrials.gov and in the grey literature source OpenGrey.eu. The RCT and quasi-RCT comparison techniques isolated or in combination for treating PVs will be considered. Three review authors will independently perform data extraction and quality assessments of data from included studies, and any disagreements will be resolved by discussion. The primary outcomes will be wound healing and pain. Secondary outcomes will include oedema, adverse events, recurrence or recanalisation, quality of life and economic aspects. The Cochrane handbook will be used for guidance. If the results are not appropriate for a meta-analysis in RevManV.5 software (eg, if the data have considerable heterogeneity and are drawn from different comparisons), a descriptive analysis will be performed.

Ethics and dissemination Ethics committee approval is not necessary. We intend to update the public registry used in this review, report any important protocol amendments and publish the results in a widely accessible journal. PROSPERO registration number CRD42018092974

\section{INTRODUCTION}

Perforator veins (PVs) play an important role in the development of chronic venous
Strengths and limitations of this study

- Well-accepted standards for the conduct and reporting of systematic reviews will be followed.

- The systematic approach (Cochrane handbook and GRADE approach) for summarising the current available evidence on the effects of various types of interventions for the treatment of incompetent perforator veins will strengthen the review.

- This review may allow future contributions to high-quality guidelines and better decision-making on the choice of treatment modality for the segment of the population with chronic venous disease, given that the range of clinical manifestations and treatment options make this type of disease a challenge.

- Since only randomised-controlled trials (RCTs) or quasi-RCTs will be included, some old comparisons (eg, with intra-operative reflux visualisation) may have little available evidence.

insufficiency and ulceration ${ }^{1}$ and are a significant factor in the determination of chronic venous disease severity. ${ }^{2}$ Since 1916 , when Homans made the first description, PVs have been implicated in the development of venous ulcers. ${ }^{3}$ Currently, there is no established preferred procedure for the treatment of pathologic perforator veins (PPVs) in patients designated as clinical class 2 and clinical class 3 of the Clinical Aetiologic Anatomic Pathophysiologic (CEAP) classification $^{4}$; however, patients with venous ulcers should receive attention for the treatment of PVs in cases of failure to treat truncal surface reflux. ${ }^{5}$ Clinical practice guidelines of the Society for Vascular Surgery (SVS) and the American Venous Forum (AVF) recommend the treatment of PVs with diameters $>3.5 \mathrm{~mm}$ and reflux time $>0.5 \mathrm{~s}$ located near healed or active venous ulcers. ${ }^{67}$ However, this recommendation is weak and, at most, obtains moderate evidence certainty because it is 
based on randomised controlled trials (RCTs) with small samples and systematic reviews (SRs) with serious methodological biases. For instance, some SRs filtered data by publication language and other SRs meta-analysed RCTs with case series without a justified reason. ${ }^{6} 7$ The most recent SVS/AVF document about the treatment of chronic venous diseases recommends the selective treatment of incompetent PVs in patients with simple varicose veins (CEAP C2). However, its recommendation is also based on small RCTs or SRs with serious methodological issues. The European Society for Vascular Surgery practice guidelines lack robust evidence assessing the treatment of PPVs but define PPVs as a reflux time greater than $0.35 \mathrm{~s}^{8}$

Procedures to eliminate incompetence and reflux in PVs may use surgical ligation, subfascial endoscopic surgery, intravenous ablation techniques and sclerotherapy. ${ }^{3}$ With improvements in technology, traditional open surgical options for the disconnection of PVs have been supplanted by minimally invasive techniques in practice, even without robust evidence. Regardless of the method used, occlusion of a PV is useful for wound healing and venous insufficiency with minimal morbidity. ${ }^{5}$ Therefore, there is an interest in comparing surgical options for the treatment of incompetent PVs. Although the rates of PV closure initially reported for percutaneous thermal ablation ranged from $60 \%$ to $80 \%$, vein recanalisation and the formation of new incompetent veins have also been reported. ${ }^{1}$ Ultrasound-guided foam sclerotherapy has shown promise in the closure of PVs and in wound healing but with varying success rates and associated possible associate adverse effects (eg, deep vein thrombosis, pulmonary embolism and death). ${ }^{9} 10$

Evidence of the benefits of saphenous ablation has been growing; however, there is no high-quality evidence that addresses the treatment of incompetent PVs. ${ }^{11-13}$ With the aim of filling the evidence gap, this is a protocol for an SR and meta-analysis that will assess the effects of any form of intervention, isolated or in combination, for the treatment of PPVs of the lower limbs in patients with chronic venous disease, a CEAP clinical classification of 0-6, with clinical and objective evidence of PPVs.

\section{METHODS}

This protocol is prospectively registered in the international prospective register of SRs PROSPERO database (CRD42018092974) and is based on the Cochrane handbook of interventions reviews. ${ }^{14}$ In addition, the Preferred Reporting Items for Systematic Review and Meta-Analysis (PRISMA) Protocols are used to report this protocol. ${ }^{15}$

\section{Types of studies}

RCTs and quasi-RCTs will be accepted; thus, other study designs will be excluded for the purpose of this review. A quasi-RCT is a trial in which participants are allocated to different groups using a method of allocation that is not truly random (eg, date of birth, medical record number or the order in which people were recruited). ${ }^{16}$

\section{Types of participants}

While there are recommendations for the treatment of PPVs in patients with CEAP C5-6, the evidence favouring PPVs treatment in those with CEAP C0-4 is more sparse. ${ }^{78}$ However, PPVs is recognised as an important risk factor for the determination of chronic venous disease severity. ${ }^{2}$ Therefore, patients with chronic venous disease, a CEAP clinical classification 0-6, with clinical and objective evidence of PPVs and without any limits on age and gender will be considered. The diagnosis of PPVs will be considered valid only with at least one of the following objective evaluations:

- PV reflux on duplex ultrasound (DUS) (ie, reflux time of at least $0.35 \mathrm{~s}$ ) with the patient in the erect position. $^{8}$

- PPVs on digital subtraction angiography (DSA) (ie, ascending or descending phlebography). ${ }^{817}$

- Intraoperative PV reflux. ${ }^{17}$

An objectively confirmed diagnosis is essential for patient inclusion. Traditional clinical tests (eg, Trendelenburg and Perthes) will not be considered adequate because they have been shown to be inaccurate and have no place in the mapping of venous incompetence in general, particularly in the case of PPVs. ${ }^{818}$ Other methods such as CT angiography, MRI or intravascular ultrasound are not routinely used for the diagnosis of PPVs and therefore will not be considered a valid method. ${ }^{8}$

\section{Types of interventions}

We will study all the following techniques, isolated or in combination, for treating PV insufficiency:

- Non-operative therapy; for example, any treatment without direct intervention on the insufficient PV (eg, local wound care, phlebotonics ${ }^{19}$ and compression therapy).

- Conventional surgery; for example, traditional treatment, which is open exposure and subfascial ligation of PPVs. ${ }^{20}$

- Subfascial endoscopic perforator surgery (SEPS) involving the endoscopic access and identification of PPVs and their ligation. ${ }^{21}$

- Minimally invasive vein surgery, which is performed percutaneously, with minimal anaesthesia, no incisions, and rarely requires hospital admission. ${ }^{22}$ There are a variety of minimally invasive techniques and treatment modalities, including ultrasound-guided sclerotherapy and endovascular thermal ablation with laser or radiofrequency energy. These techniques can be performed in an office setting with local anaesthesia. ${ }^{23}$

We will consider all of the following possible comparisons:

- Minimally invasive vein surgery (all types) versus conventional surgery.

- SEPS versus conventional surgery. 
- Non-operative therapy versus conventional surgery.

- Any combination of the above treatments versus any combination.

\section{Types of outcome measures}

Following the Cochrane recommendations, all the outcomes will be meaningful for decision makers, that is, clinicians, patients, the general public, administrators and policy makers. ${ }^{24}$ Almost $80 \%$ of the general population may suffer from CEAP C1, and 30\% may suffer from CEAP C2. Pain is the main symptom in venous disease, including PPVs. Pain is also a well-known contributor to decreasing quality of life. Only $1 \%-2 \%$ of these patients may develop an ulcer (CEAP C6). ${ }^{8}{ }^{25}$ While the treatment for PPVs also aims to reduce pain, all types of these surgical interventions may inflict pain as an adverse event. Therefore, pain and ulcer healing are the primary outcomes, and all other patient-relevant outcomes are listed as secondary.

\section{Primary outcomes}

- Wound healing, assessed by any valid objective method as time to complete healing, proportion of ulcers healed within the trial period, change in ulcer size (surface area or volume) or rate of change in ulcer size (surface area or volume) ${ }^{26}$

- Pain, assessed as a continuous outcome (eg, mean change in pain, measured by visual analogue, numerical or categorical scale) or as a dichotomous outcome (eg, proportion of participants reporting complete pain relief or proportion reporting at least $30 \%$ [moderate] or $50 \%$ [substantial] improvement in pain).

\section{Secondary outcomes}

- Oedema, assessed as a continuous outcome (eg, mean change in oedema, measured on a numerical or categorical scale and by circumference or diameter of the limb), or as a dichotomous outcome (eg, proportion of participants with complete oedema relief or proportion with at least $50 \%$ improvement in oedema).

- Adverse events including major (eg, anaphylaxis, venous thromboembolism and death) and minor adverse events (eg, haematoma, hyperpigmentation, bruising, necrosis of the skin and superficial thrombophlebitis) will be considered.

- Recurrence or recanalisation, assessed by an objective method (DUS or DSA).

- Quality of life, assessed by any validated scale (eg, Venous Insufficiency Epidemiological and Economic Studies (VEINES-QoL), ${ }^{27}$ Aberdeen Varicose Vein Severity Score (AVVSS) ${ }^{28}$ or Short Form Health Survey 36 (SF-36). ${ }^{29} 30$

- Economic aspects, assessed by any available data (eg, duration of hospital stay, cost of hospital stay or cost associated with intervention).

\section{Search methods}

\section{Electronic searches}

Systematic searches will be carried out, at least, in the following databases: Medical Literature Analysis and Retrieval System Online (MEDLINE [via PubMed]), Excerpta Medica database (EMBASE [via Elsevier]), Cochrane Central Register of Controlled Trials (CENTRAL [via Wiley]), Indice Bibliográfico Español de Ciencias de la Salud (IBECS) and Latin American and Caribbean Literature on Health Sciences (LILACS) (both via Virtual Health Library). The search strategy will consist of controlled terms (eg, MeSH, Emtree) and free-text terms related to 'varicose veins', 'veins', 'perforator vein', 'venous insufficiency', 'laser therapy', 'laser coagulation', 'subfascial endoscopic perforator surgery', 'endoscopy', 'sclerosing solutions', 'vein stripping' and 'endovascular procedures'. No limits on language, date or status of the publication will be imposed. Beyond that, a search will also be carried out in the WHO International Clinical Trials Registry Platform, in the clinical trial registries of ClinicalTrials.gov, and in the grey literature source OpenGrey.eu. The electronic search strategies will be the most sensitive possible. ${ }^{14}$ A sample search strategy for MEDLINE via PubMed is presented in table 1.

\begin{tabular}{|c|c|}
\hline Line & Search \\
\hline 1 & $\begin{array}{l}\text { 'Varicose Veins'[Mesh] or (Varicose Vein*) or (Varix) or } \\
\text { (Varices) }\end{array}$ \\
\hline 2 & ‘Veins'[Mesh] or (Vein*) or (perforator vein*) \\
\hline 3 & 'Venous Insufficiency'[Mesh] or (Insufficienc* Venous) \\
\hline 4 & $\begin{array}{l}\text { 'Laser Therapy'[Mesh] or (Laser Therap*) or } \\
\text { (Vaporization Laser) or (Laser Ablation) or (Laser } \\
\text { Tissue Ablation) or (Pulsed Laser Tissue Ablation) or } \\
\text { (Laser Photoablation of Tissue) or (Nonablative Laser } \\
\text { Treatment*) or (Laser Scalpel*) or (Laser Knive }) \text { or } \\
\left.(\text { Laser Knife }) \text { or (Laser Surger }{ }^{\star}\right)\end{array}$ \\
\hline 5 & $\begin{array}{l}\text { 'Laser Coagulation'[Mesh] or (Thermocoagulation* } \\
\text { Laser) or (Coagulation* Laser) }\end{array}$ \\
\hline 6 & $\begin{array}{l}\text { ‘Endoscopy’[Mesh] or (Surgical Procedure* } \\
\text { Endoscopic) or (Endoscopy Surgical) or (Endoscopic } \\
\text { Surgical Procedure*) }\end{array}$ \\
\hline 7 & $\begin{array}{l}\text { 'Sclerosing Solutions'[Mesh] or (Solution* Sclerosing) } \\
\text { or (Sclerosing Injection*) or }\left(\text { Sclerosing Agent }{ }^{\star}\right) \text { or } \\
\left.\text { (Sclerosant }^{*}\right) \text { or }\left(\text { Sclerosing Drug*) }^{*}\right.\end{array}$ \\
\hline 8 & $\begin{array}{l}\text { 'Endovascular Procedures'[Mesh] or (Endovascular } \\
\text { Procedure*) or (Intravascular Procedure }) \text { or } \\
\text { (Intravascular Technique }^{\star} \text { ) or (Endovascular } \\
\text { Technique }{ }^{\star} \text { ) }\end{array}$ \\
\hline 9 & $\begin{array}{l}\text { (subfascial endoscopic perforator surgery) or (vein } \\
\text { stripping) or (stripping) or SEPS }\end{array}$ \\
\hline 10 & 1 or 2 or 3 \\
\hline 11 & 4 or 5 or 6 or 7 or 8 or 9 \\
\hline 12 & 10 and 11 \\
\hline 13 & Search (Therapy/Broad[filter]) AND (\#12) \\
\hline
\end{tabular}




\section{Manual search}

The reference lists of all included studies and review articles will be searched for additional RCTs or quasiRCTs. Manufacturers and specialists in the field of phlebology will be contacted, mainly for further unpublished research. In addition, authors of identified trials will be asked about additional data from the included trials as well as for other additional studies.

\section{Selection of studies}

Three independent reviewers (MAMS, RLGF and LCUN) will evaluate the trials to determine if they are appropriate for inclusion. Any disagreement will be solved by discussion with the author's team (FMJ, RSC, SGJS and HJGN). Full-text articles of potentially eligible studies will be analysed in detail, and then it will be decided which ones will be included; the reasons for study exclusion will be presented. Sufficient details on the selection process will be recorded, and the PRISMA flow diagram and tables will be filled in with the characteristics of the included and excluded studies. ${ }^{15}$

\section{Data extraction and management}

The following data on the study characteristics and outcomes of the included studies will be extracted by three independent reviewers (MAMS, RLGF and LCUN) ${ }^{14}$ :

- Methods: study design, total duration of the study and period of carryout, number and location of study centres, research setting, withdrawals and date of study. Participants: number, age parameters (ie, mean, range and so on), gender, the severity of the condition, diagnostic criteria and inclusion/exclusion criteria.

- Interventions: intervention, comparison, concomitant medications, and excluded medications.

- Outcomes: primary and secondary outcomes (the final outcomes reported and those planned) and time points reported.

- Notes: funding for trial and notable conflicts of interest of trial authors.

One author (MAMS) will enter these data into Review Manager 5 software (RevMan 5), version 5.3, for statistical analysis. ${ }^{31}$ If the results are not appropriate for a meta-analysis, a descriptive analysis will be performed.

\section{Assessment of risk of bias in included studies}

Three independent reviewers (MAMS, RLGF and LCUN) will assess the following risk of bias domains: (1) random sequence generation, (2) allocation concealment, (3) blinding of participants and personnel, (4) blinding of outcome assessment, (5) incomplete outcome data, (6) selective outcome reporting and (7) other bias. Each of these domains will be graded as high, low or unclear risk of bias according to the Cochrane handbook. ${ }^{14}$ Blinding will be considered separately for different key outcomes when necessary. When considering treatment effects, the risk of bias of the studies that contributed to that outcome will be taken into account.

\section{Measures of treatment effect}

Dichotomous data will be analysed as risk ratio and continuous data with the same scales as the mean difference. A standardised mean difference will be used for continuous data with different scales. CI of $95 \%$ will be considered for all data.

\section{Unit of analysis issues}

Lower limbs will be the unit of analysis for all outcomes (eg, ulcer healing). Nevertheless, the participant will be the unit of analysis for other outcomes such as pain, mortality, adverse events, quality of life and length of hospitalisation. The intention-to-treat approach will be used.

\section{Addressing missing data}

We will contact the authors or sponsors of the included studies, either to verify details about methods and characteristics or to request missing numerical outcome data. Significant bias is not expected if both intervention groups have outcome data missing with reasons that are balanced and reported across groups. However, if the missing data have different implications in the compared groups, the studies will be considered to have a high risk of bias. The frequency (or risk) of outcomes has a direct influence on the potential impact of missing data in dichotomous outcomes studies, while the proportion of participants with missing data is directly related to the potential impact on continuous outcomes studies. ${ }^{14}$

\section{Assessment of heterogeneity}

The studies will be assessed for methodological and clinical heterogeneity using the $\mathrm{I}^{2}$ statistic (where $\mathrm{I}^{2}=\left[\left(\mathrm{Q}^{-}\right.\right.$ df) $/ Q]$ x $100 \%$; $Q=X^{2}$ statistic; 'df'=degree of freedom). Meta-analyses will be conducted if, after these analyses, the studies are considered to have acceptable heterogeneity. We will analyse statistical heterogeneity by visual inspection of the forest plots if possible (ie, if more than 10 studies are included). As strict thresholds for interpretation of $\mathrm{I}^{2}$ are not recommended, we will follow the rough guide for interpretation in the Cochrane handbook for SRs of interventions ${ }^{32}$ :

- $0 \%$ to $40 \%$ : possibly not important.

- $30 \%$ to $60 \%$ : may represent moderate heterogeneity.

- $50 \%$ to $90 \%$ : may represent substantial heterogeneity.

- $75 \%$ to $100 \%$ : considerable heterogeneity.

Heterogeneity stratification demonstrates the percent variability in the estimated effects resulting from heterogeneity rather than sampling error. ${ }^{14}$ We will attempt to explain any substantial heterogeneity by performing subgroup analyses on the pre-specified groups and by examining the findings of the evaluation of publication bias.

\section{Assessment of reporting biases}

If there is an adequate number of studies (at least 10) for inclusion in a meta-analysis, the presence of publication bias and other types of reporting bias will be assessed using funnel plots. ${ }^{1433} 34$ 


\section{Data synthesis}

We will synthesise qualitative information relative to methods, risk of bias, description of participants, and outcome measures and insert this information in the 'Characteristics of included studies' tables using the software RevMan 5, and a meta-analysis will be performed whenever it is feasible. ${ }^{31}$ We will not include qualitative (non-randomised) studies in the review. We will use a fixed-effects model in a meta-analysis with very homogenous included studies, considering population, interventions, comparators and outcomes characteristics. Otherwise, we will use a random-effects model if at least substantial heterogeneity ( $\mathrm{I}^{2}$ of $50 \%$ or more) is identified, or if significant clinical differences regarding patients and interventions exist among included trials. ${ }^{32}$

\section{Subgroup analysis and investigation of heterogeneity}

In the case of substantial heterogeneity, we will perform a subgroup analysis to explore possible causes. It will be carried out for participants' characteristics (eg, age, gender and race) and intervention characteristics (eg, type of treatment). We will attempt to contact the authors to obtain missing data when data are not available from the original publications.

\section{Sensitivity analysis}

A sensitivity analysis will be conducted to determine the impact of exclusion of quasi-RCTs or studies with an overall high risk of bias, which are those studies with a high risk of bias in at least one of the main domains in the risk of bias tool (generation of randomisation sequence, allocation concealment and blinding). ${ }^{32}$

\section{'Summary of findings' table}

We will use the Grading of Recommendations Assessment, Development and Evaluation (GRADE) software (GRADEpro) to generate a 'Summary of findings' table for each one of the outcomes to be analysed in this review. ${ }^{35}$ Using the five GRADE criteria (study limitations, consistency of effect, imprecision, indirectness and publication bias), we will assess the quality of the body of evidence that made up the data for the meta-analyses of the pre-specified outcomes. ${ }^{35}{ }^{36}$ These criteria will be evaluated, and the table will be filled in by using the Cochrane recommendations, justifying any departures from the standard methods. ${ }^{14}$

\section{Patient and public involvement}

The research question was developed from the authors' experience treating patients with venous disease, associated with methodological knowledge and under the advisement of a patient group for the selection of the main patient-relevant outcomes. Patients were involved in the design of this protocol in the form of a discussion group to define the research question, for outcome selection and to ensure comprehension of the final manuscript. However, they did not contribute sufficiently to qualify as co-authors. We intend to include patients in all steps of this research as advisors and to maintain comprehensive language in the final text that will be appropriate for consumers. The final version of this review, with results, conclusions and any changes in the protocol, will be published in an accessible international journal; presented in related congresses, conferences and meetings; and sent to the offices of health policy makers.

\section{Ethics and dissemination}

We will not treat patients directly; therefore, ethics committee approval is not necessary because it is not a primary study. It is hoped that the authors of primary studies to be included in the analysis have already obtained such approval. We intend to update the public registry with this review in all phases of its execution, report any important protocol amendments and publish the results in a widely accessible journal.

Correction notice This article has been corrected since it was published online. Orcid lds have been updated for the authors.

Acknowledgements The authors would like to thank the patient advisers, Cochrane Brazil, Brazilian Society of Angiology and Vascular Surgery—São Paulo section and the Division of Vascular and Endovascular Surgery, Universidade Federal de São Paulo, Brazil, for their support.

Contributors MAMS: coordinated contributions from coauthors, drafted the clinical sections, contributed to writing the protocol, will perform future review updates and is the guarantor of the review. RLGF: worked on the clinical and methods sections, contributed to writing the protocol and will perform future review updates. FMJ, RSC, SGJS, HJGN and LCUN: worked on the methods sections and contributed to writing the protocol. All authors have read and approved the final version of the manuscript.

Funding The authors have not declared a specific grant for this research from any funding agency in the public, commercial or not-for-profit sectors.

Competing interests None declared.

Patient consent for publication Not required.

Provenance and peer review Not commissioned; externally peer reviewed.

Open access This is an open access article distributed in accordance with the Creative Commons Attribution Non Commercial (CC BY-NC 4.0) license, which permits others to distribute, remix, adapt, build upon this work non-commercially, and license their derivative works on different terms, provided the original work is properly cited, appropriate credit is given, any changes made indicated, and the use is non-commercial. See: http://creativecommons.org/licenses/by-nc/4.0/.

\section{REFERENCES}

1. Aurshina A, Hingorani A, Blumberg S, et al. Shortened protocol for radiofrequency ablation of perforator veins. J Vasc Surg Venous Lymphat Disord 2017;5:824-8.

2. Delis KT. Perforator vein incompetence in chronic venous disease: a multivariate regression analysis model. J Vasc Surg 2004;40:626-33.

3. Lawrence PF, Alktaifi A, Rigberg D, et al. Endovenous ablation of incompetent perforating veins is effective treatment for recalcitrant venous ulcers. J Vasc Surg 2011;54:737-42.

4. Eklöf B, Rutherford RB, Bergan JJ, et al. Revision of the CEAP classification for chronic venous disorders: consensus statement. J Vasc Surg 2004;40:1248-52.

5. Dillavou ED, Harlander-Locke M, Labropoulos N, et al. Current state of the treatment of perforating veins. J Vasc Surg Venous Lymphat Disord 2016;4:131-5.

6. O'Donnell TF, Passman MA, Marston WA, et al. Management of venous leg ulcers: clinical practice guidelines of the Society for

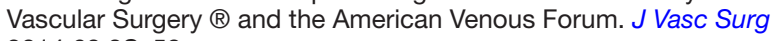
2014;60:3S-59.

7. Gloviczki P, Comerota AJ, Dalsing MC, et al. The care of patients with varicose veins and associated chronic venous diseases: clinical practice guidelines of the Society for Vascular Surgery and the American Venous Forum. J Vasc Surg 2011;53:2S-48. 
8. Wittens C, Davies AH, Bækgaard N, et al. Editor's Choice Management of Chronic Venous Disease: Clinical Practice Guidelines of the European Society for Vascular Surgery (ESVS). Eur J Vasc Endovasc Surg 2015;49:678-737.

9. Bush R, Bush P. Percutaneous foam sclerotherapy for venous leg ulcers. J Wound Care 2013;22:S20-S22.

10. Campos W, Torres IO, da Silva ES, et al. A prospective randomized study comparing polidocanol foam sclerotherapy with surgical treatment of patients with primary chronic venous insufficiency and ulcer. Ann Vasc Surg 2015;29:1128-35.

11. Samuel N, Carradice D, Wallace T, et al. Endovenous thermal ablation for healing venous ulcers and preventing recurrence. Cochrane Database Syst Rev 2013:CD009494.

12. Nesbitt C, Bedenis R, Bhattacharya V, et al. Endovenous ablation (radiofrequency and laser) and foam sclerotherapy versus open surgery for great saphenous vein varices. Cochrane Database Syst Rev 2014:CD005624.

13. Paravastu SC, Horne M, Dodd PD. Endovenous ablation therapy (laser or radiofrequency) or foam sclerotherapy versus conventional surgical repair for short saphenous varicose veins. Cochrane Database Syst Rev 2016;11:CD010878.

14. Higgins J, Churchill D, Chandler J, et al. Cochrane Handbook for Systematic Reviews of Interventions version 5.2.0 (updated June 2017). 2017 https://training.cochrane.org/handbook/pdf-versions (Accessed 28 Feb 2018).

15. Moher D, Shamseer L, Clarke M, et al. Preferred reporting items for systematic review and meta-analysis protocols (PRISMA-P) 2015 statement. Syst Rev 2015;4:1.

16. Lefebvre C, Manheimer E, Glanville J. Chapter 6: Searching for studies. In: Higgins JPT, Green S, eds. Cochrane Handbook of Systematic Reviews of Intervention. Version 5.1.0 (updated March 2011), 2011.

17. Meyer T, Cavallaro A, Lang W. Duplex ultrasonography in the diagnosis of incompetent Cockett veins. Eur J Ultrasound 2000;11:175-80.

18. Kim J, Richards S, Kent PJ. Clinical examination of varicose veins--a validation study. Ann R Coll Surg Engl 2000;82:171-5.

19. Martinez-Zapata MJ, Vernooij RW, Uriona Tuma SM, et al. Phlebotonics for venous insufficiency. Cochrane Database Syst Rev 2016;4:CD003229.

20. Linton RR. The communicating veins of the lower leg and the operative technic for their ligation. Ann Surg 1938;107:582-93.

21. Gloviczki P, Bergan JJ, Rhodes JM, et al. Mid-term results of endoscopic perforator vein interruption for chronic venous insufficiency: lessons learned from the North American subfascial endoscopic perforator surgery registry. The North American Study Group. J Vasc Surg 1999;29:489-502.

22. Poblete H, Elias S. Venous ulcers: new options in treatment: minimally invasive vein surgery. J Am Col Certif Wound Spec 2009:1:12-19.

23. Kuyumcu G, Salazar GM, Prabhakar AM, et al. Minimally invasive treatments for perforator vein insufficiency. Cardiovasc Diagn Ther 2016;6:593-8.

24. Green S, Higgins J. Chapter 2: Preparing a Cochrane review. In: Higgins JPT, Green S, eds. Cochrane Handbook of Systematic Reviews of Intervention. Version 5.1.0 (updated March 2011), 2011.

25. Onida S, Davies AH. Predicted burden of venous disease. Phlebology 2016:31:74-9.

26. O'Meara S, Cullum N, Nelson EA, et al. Compression for venous leg ulcers. Cochrane Database Syst Rev 2012;11:CD000265.

27. Bland JM, Dumville JC, Ashby RL, et al. Validation of the VEINESQOL quality of life instrument in venous leg ulcers: repeatability and validity study embedded in a randomised clinical trial. BMC Cardiovasc Disord 2015;15:85.

28. Smith JJ, Garratt AM, Guest M, et al. Evaluating and improving health-related quality of life in patients with varicose veins. $J$ Vasc Surg 1999;30:710-9.

29. Ware JE, Sherbourne CD. The MOS 36-item short-form health survey (SF-36). I. Conceptual framework and item selection. Med Care 1992;30:473-83.

30. Ciconelli R, Ferraz M, Santos W, et al. Tradução para a língua portuguesa e validação do questionário genérico de avaliação de qualidade de vida SF-36 (Brasil SF-36). Rev Bras Reumatol 1999;39:143-50.

31. Review Manager 5 (RevMan 5) [Computer program]. Version 5.3. Copenhagen: Nordic Cochrane Centre, Cochrane, 2014

32. Deeks JJ, Higgins J, Altman D, et al. et alChapter 9: Analysing data and undertaking meta-analyses. In: Higgins JPT, Churchill R, Chandler J, Cumpston MS, . eds. Cochrane Handbook for Systematic Reviews of Interventions version 5.2.0 (updated June 2017), Cochrane, 2017.

33. Higgins JP, Thompson SG, Deeks JJ, et al. Measuring inconsistency in meta-analyses. BMJ 2003;327:557-60.

34. Higgins JP, Whitehead A, Simmonds M. Sequential methods for random-effects meta-analysis. Stat Med 2011;30:903-21.

35. GRADEpro GDT. GRADEpro. Hamilton, Ontario, Canada: McMaster University and Evidence Prime Inc, 2015.

36. Guyatt GH, Oxman AD, Vist GE, et al. GRADE: an emerging consensus on rating quality of evidence and strength of recommendations. BMJ 2008;336:924-6. 